\title{
Evaluation of the interrelations between thyroid function, insulin resistance, lipid profile, C-reactive protein and homocysteine in patients with autoimmune thyroiditis
}

Celestino Neves ${ }^{1}$, João Sérgio Neves ${ }^{1}$, Sofia Castro Oliveira ${ }^{1}$, César Esteves $^{1}$, Oksana Sokhatska ${ }^{2}$, Camila Dias $^{3}$, Luís Delgado², José Luís Medina ${ }^{1}$ \& Davide Carvalho ${ }^{1,4}$

\begin{abstract}
${ }^{1}$ Department of Endocrinology, Diabetes and Metabolism, São João Hospital Center; Faculty of Medicine of the University of Porto (FMUP), Porto, Portugal; ${ }^{2}$ Immunology Department, São João Hospital, Faculty of Medicine of University of Porto, Porto, Portugal; ${ }^{3}$ Department of Health Information and Decision Sciences, São João Hospital, Faculty of Medicine of University of Porto, Porto, Portugal; ${ }^{4}$ Instituto de Investigação e Inovação em Saúde, University of Porto, Porto, Portugal.
\end{abstract}

\section{Introduction}

In patients with autoimmune thyroiditis, thyroid function appears to be related to increased cardiovascular risk.

\section{Objective}

It was our objective to evaluate the relationship between TSH, insulin resistance, lipid profile, $\mathrm{C}$-reactive protein (CRP) and homocysteine in patients with autoimmune thyroiditis (AIT).

\section{Methods}

- We assessed medical records of $\mathbf{2 4 2}$ patients with autoimmune thyroiditis from consultation of Endocrinology.

- Patients with other autoimmune diseases, diabetes mellitus, oncologic pathology, previous cardiovascular events and taking medication to dyslipidemia or thyroid disease were excluded.

- We defined 2 groups:

- Group 1 ( $n=171)$ - euthyroid state: TSH 0.35-2.0 $\mu \mathrm{UI} / \mathrm{ml}$, fT3 $1.71-3.71 \mathrm{pg} / \mathrm{ml}$ and fT4 0.70-1.48 ng/dl

- Group 2 ( $n=71$ ) - subclinical hypothyroidism: TSH> $2.0 \mathrm{UI} / \mathrm{ml}$, fT3 $1.71-3.71 \mathrm{pg} / \mathrm{ml}$ and fT4 $0.70-1.48 \mathrm{ng} / \mathrm{dl}$

- We recorded thyroid function tests, anti-TPO and antithyroglobulin antibodies, total cholesterol, LDL-cholesterol, HDL-cholesterol, triglycerides, apolipoproteinA1, apolipoproteinB, lipoprotein(a), homocysteine, high sensitivity CRP, folic acid, vitamin B12, HOMA-IR (Homeostasis model assessment insulin resistance), HOMA- $\beta$ (Homeostasis model assessment $\beta$ cell), QUICKI (Quantitative insulin sensitivity check index), HISI (Hepatic insulin sensitivity index), WBISI (Whole body insulin sensitivity index) and IGI (Insulinogenic index) for both groups.

- Statistical analysis was performed with Mann-Whitney test, logistic regression and Spearman correlations. Statistical significance was considered for a bilateral value of $p<0.05$.

\section{Results}

- We found significantly higher levels of insulin at $120 \mathrm{~min}$ of OGTT

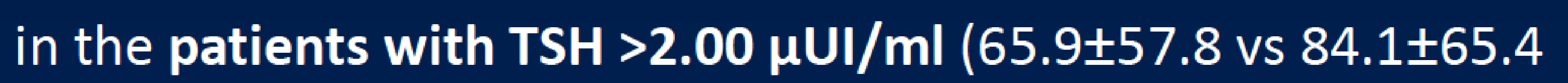
$\mu \mathrm{UI} / \mathrm{ml} ; \mathrm{P}=0.02)$.

- The levels of homocysteine were also significantly higher in the group with TSH $>2.00 \mu \mathrm{Ul} / \mathrm{ml}(10.8 \pm 12.6$ vs $8.3 \pm 3.3 \mu \mathrm{mol} / \mathrm{l} ; \mathrm{P}=0.04)$.

- We found the IGI $(0.036 \pm 0.378$ vs $0.252 \pm 0.310 ; P=0.02)$ and WBISI (6.323 \pm 7.335 vs $6.112 \pm 4.019 ; P=0.003)$ indexes to be significantly higher in the TSH $>2.00 \mu \mathrm{UI} / \mathrm{ml}$ group.
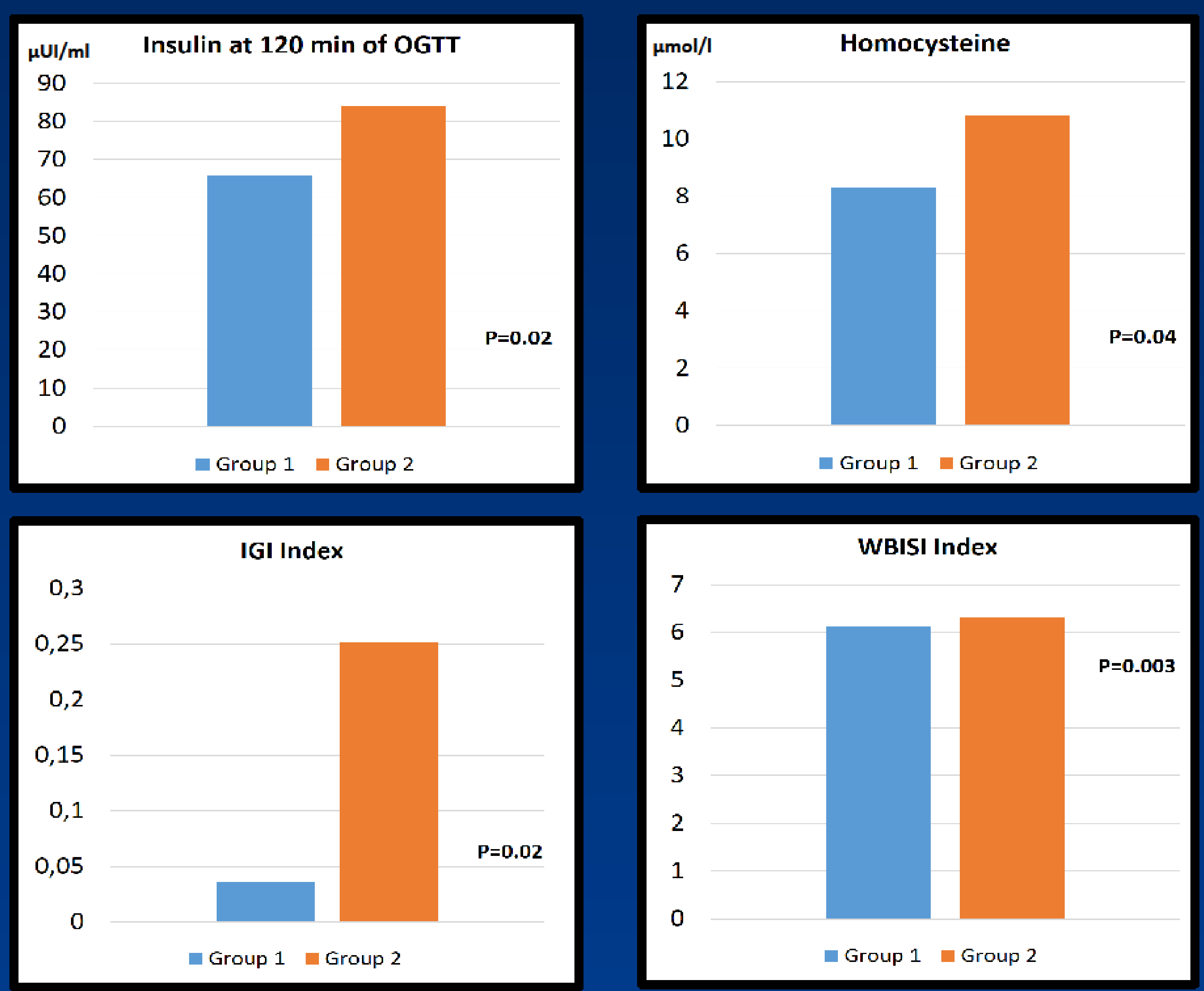

\section{- Spearman correlations:}

- Group 1 (TSH 0.35-2.0 UI/ml):

- In the group with TSH $<2.00 \mu \mathrm{UI} / \mathrm{ml}$ there were positive correlations between IGI and the triglyceride levels $(r=0.256 ; P=0.004)$ and the anti-TPO levels $(r=0.137 ; P=0.03)$.

- In the same group we found negative correlations between WBISI and CRP ( $r=-0.199 ; P=0.02$ ) and positive correlations between WBISI and TSH $(r=0.44 ; P=0.01)$.

- Group 2 (TSH > 2.0 UI/ml):

- In the group with TSH $>2.00 \mu \mathrm{Ul} / \mathrm{ml}$ we found positive correlations between the FT4 levels and the BMI $(r=0.413 ; P<0.001)$.

- In the same group the levels of LDL were positively correlated with TSH ( $r=0.245 ; P=0.04)$, and negatively with FT3 ( $r=-0.265 ; P=0.02)$.

- There was also a positive correlation between the $L p(a)$ and FT4 levels ( $r=0.259 ; P=0.04)$.

\section{Conclusions}

In patients with AIT, the relationship between thyroid function, lipid profile, homocysteine and the insulin resistance indexes, may contribute to an increased cardiovascular risk 